\title{
Medication patterns in older adults with multimorbidity: a cluster analysis of primary care patients
}

\author{
Marina Guisado-Clavero 1,2,3, Concepción Violán ${ }^{2,3^{*}}$ (D), Tomàs López-Jimenez ${ }^{2,3}$, Albert Roso-Llorach, \\ Mariona Pons-Vigués ${ }^{2,3,4}$, Miguel Angel Muñoz ${ }^{1,2,3,5}$ and Quintí Foguet-Boreu $2,3,6,7$
}

\begin{abstract}
Background: Older adults suffer from various chronic conditions which make them particularly vulnerable. The proper management of multiple drug use is therefore crucial. The aim of our study was to describe drug prescription and medication patterns in this population.

Methods: A cross-sectional study in Barcelona (Spain) using electronic health records from 50 primary healthcare centres. Participants were aged 65 to 94 years, presenting multimorbidity ( $\geq 2$ chronic diseases), and had been prescribed at least 1 drug for 6 months or longer during 2009. We calculated the prevalence of prescribed drugs and identified medication patterns using multiple correspondence analysis and k-means clustering. Analyses were stratified by sex and age (65-79, 80-94 years).

Results: We studied 164,513 patients (66.8\% women) prescribed a median of 4 drugs (interquartile range $[\mathrm{IQR}]=3-7)$ in the 65-79 age-group and 6 drugs $(\mathrm{IQR}=4-8)$ in the $80-94$ age-group. A minimum of $45.9 \%$ of patients aged $65-79$ years, and $61.8 \%$ of those aged $80-94$ years, were prescribed 5 or more drugs. We identified 6 medication patterns, a non-specific one and 5 encompassing 8 anatomical groups (alimentary tract and metabolism, blood, cardiovascular, dermatological, musculo-skeletal, neurological, respiratory, and sensory organ).

Conclusions: Drug prescription is widespread among the elderly. Six medication patterns were identified, 5 of which were related to one or more anatomical group, with associations among drugs from different systems. Overall, guidelines do not accurately reflect the situation of the elderly multimorbid, new strategies for managing multiple drug uses are needed to optimize prescribing in these patients.
\end{abstract}

Keywords: Ageing, Cluster analysis, Drugs, Electronic health records, Multimorbidity, Primary health care

\section{Introduction}

Worldwide, individuals are living longer [1] thanks to advances in medical research and care [2]. For instance, in 2016, 19\% of the European population was aged 65 years or older [3], a figure that is expected to reach $30 \%$ by 2060 [4]. Nevertheless, a longer life span is closely related to the likelihood of developing chronic disease [5] and 55-98\% of older adults suffer from multimorbidity [6]. Such patients

\footnotetext{
* Correspondence: cviolan@idiapjgol.org; http://www.idiapjgol.org/

${ }^{2}$ Institut Universitari d'Investigació en Atenció Primària Jordi Gol (IDIAP Jordi Gol), Gran Via Corts Catalanes, 587 àtic, 08007 Barcelona, Spain

${ }^{3}$ Universitat Autònoma de Barcelona, Plaça Cívica, 08193 Bellaterra, Barcelona, Spain

Full list of author information is available at the end of the article
}

are more likely to require multiple drugs to achieve optimal clinical (or disease) management [7, 8], indeed, a prescription rate of over $80 \%$ for $\geq 5$ drugs has been reported [9]. Multiple drug use in older adults, however, is associated with overall worsening physical and psychological health as a result of age-related changes in pharmacokinetics and pharmacodynamics [10]. In addition, it has a potential influence on aspects of safety, including inappropriate prescription, adverse drug reaction, risk of medication interaction (drug-drug or drug-disease interaction), and adherence $[11,12]$.

Due to ageing vulnerability, multiple drug use in the multimorbid elderly is a main issue of concern for the

(c) The Author(s). 2019 Open Access This article is distributed under the terms of the Creative Commons Attribution 4.0 International License (http://creativecommons.org/licenses/by/4.0/), which permits unrestricted use, distribution, and reproduction in any medium, provided you give appropriate credit to the original author(s) and the source, provide a link to the Creative Commons license, and indicate if changes were made. The Creative Commons Public Domain Dedication waiver (http://creativecommons.org/publicdomain/zero/1.0/) applies to the data made available in this article, unless otherwise stated. 
public health system. Identifying which drugs are being taken is crucial to define patients at risk. As a result, tools need to be developed with the aim of decreasing prescription errors, drugs interactions, adverse drug reactions, and other consequences such as falls, hospitalization, and mortality associated with multiple drug use [13, 14]. A recent systematic review described clinical management oriented to multimorbidity and polymedication. Its recommendations, however, were focused on the risks/benefits of each drug individually rather than collectively [15]. To date, the limited information available in the literature is mostly descriptive [16] and methods regarding pharmacoepidemiology in multimorbidity have yet to be established. Prescription groups and patterns could be of help in the analysis of multiple drug use to create new strategies in the management of complexity among multimorbid patients.

New techniques are being developed to create homogeneous patterns regarding the management of prescribed drugs. For instance, exploratory factor analysis (EFA) which is based on correlations between variables or factors, and cluster analysis (CA), a technique for grouping a set of individuals in such a way that they are more similar to each other than those in other groups [17]. EFA has recently been reported to be useful for describing correlation between variables, while CA carries out an in-depth examination of the pattern for non-random associations between the determinant variables of an individual [18]. In recent years, EFA has been employed to define a number of multimorbidity patterns [19-21], and some medication ones [22]. Nonetheless, the statistical technique employed should be taken into account. EFA correlates specific variables (e.g. diseases), but not all the variables of one unit (e.g. patient), whilst CA could be helpful as the main starting point to look for dissimilarities. Irrespective of the methodology employed in these studies [23], there are common biological systems encompassing multimorbidity patterns: cardio-metabolic conditions, musculoskeletal diseases, and mental health problems [24]. Serious diseases and those with a greater prevalence according to EFA/CA should thus be represented with the corresponding medication.

We hypothesized that prescribed drugs could be grouped using CA to identify clusters of patients with similar drugs and consequently create medication patterns. The objective of this study was to describe prescribed drugs and identify medication patterns in multimorbid older adults.

\section{Methods}

\section{Design, setting, and inclusion criteria}

We conducted a cross-sectional analysis of electronic health records (EHR) from the Information System for Research in Primary Care (SIDIAP). This is a centralized database that contains EHR from 2006 for all the patients who have attended primary health care centres
(PHCC) run by the public Catalan Health Institute [25, 26]. The study was performed in Barcelona (Spain) in 2009 with information from 50 PHCC. The participants were aged 65 to 94 years, and the inclusion criteria were a) to have attended a PHCC at least once during 2009; b) to present multimorbidity, defined as the coexistence of 2 or more chronic diseases [27]; and c) to have been prescribed at least 1 drug for a period of 6 months or longer during 2009 (see flow chart in Fig. 1).

The study protocol was approved by the Research Ethics Committee at IDIAPJGol (Protocol no: P15/149). All data were anonymized, and the confidentiality of the EHR was maintained at all times in accordance with national and international law. As all data were anonymized, no consent to individuals were required.

\section{Variables}

Prescription drugs were the main unit of measurement and were coded as 1 (present) or 0 (absent). Drugs in the SIDIAP database are classified using the Anatomical Therapeutic Chemical (ATC) system (Additional file 1), a measuring unit recommended by the World Health Organization for drug studies. To classify the drugs in this study, and facilitate subsequent analysis and interpretation, we used the 4th level of the ATC system which corresponds to chemical subgroups. Proton pump inhibitors, for example, are coded as A02BC [28].

The other variables recorded for each participant were: number of chronic diseases coded with the International Classification of Primary Care second edition and selected using the O'Halloran criteria [29], age (65-79 years vs $80-94$ years), and sex (male vs female). According to the chronic diseases selected, chronic medication was defined as the prescription of a drug for at least 6 continuous months during the period of study. Medication which did not fulfil this criterion was not analysed as it was considered acute or not long-term. Neither were supplements included as they are not financed by the Spanish health system.

\section{Statistical analysis}

Data were extracted from the SIDIAP database after authorization of the study [25]. All the authors had access to the database. There were no missing values, as sex, age, chronic diseases, and drugs were recorded for all the sample.

Descriptive statistics were employed to summarize the overall data. Categorical variables were expressed as frequencies (percentage) and continuous variables as means (standard deviation $[\mathrm{SD}]$ ) or medians (interquartile range [IQR]). Prevalence of prescription drugs was calculated and medication patterns identified through 2 steps: 1) multiple correspondence analysis (MCA), and 2) k- 


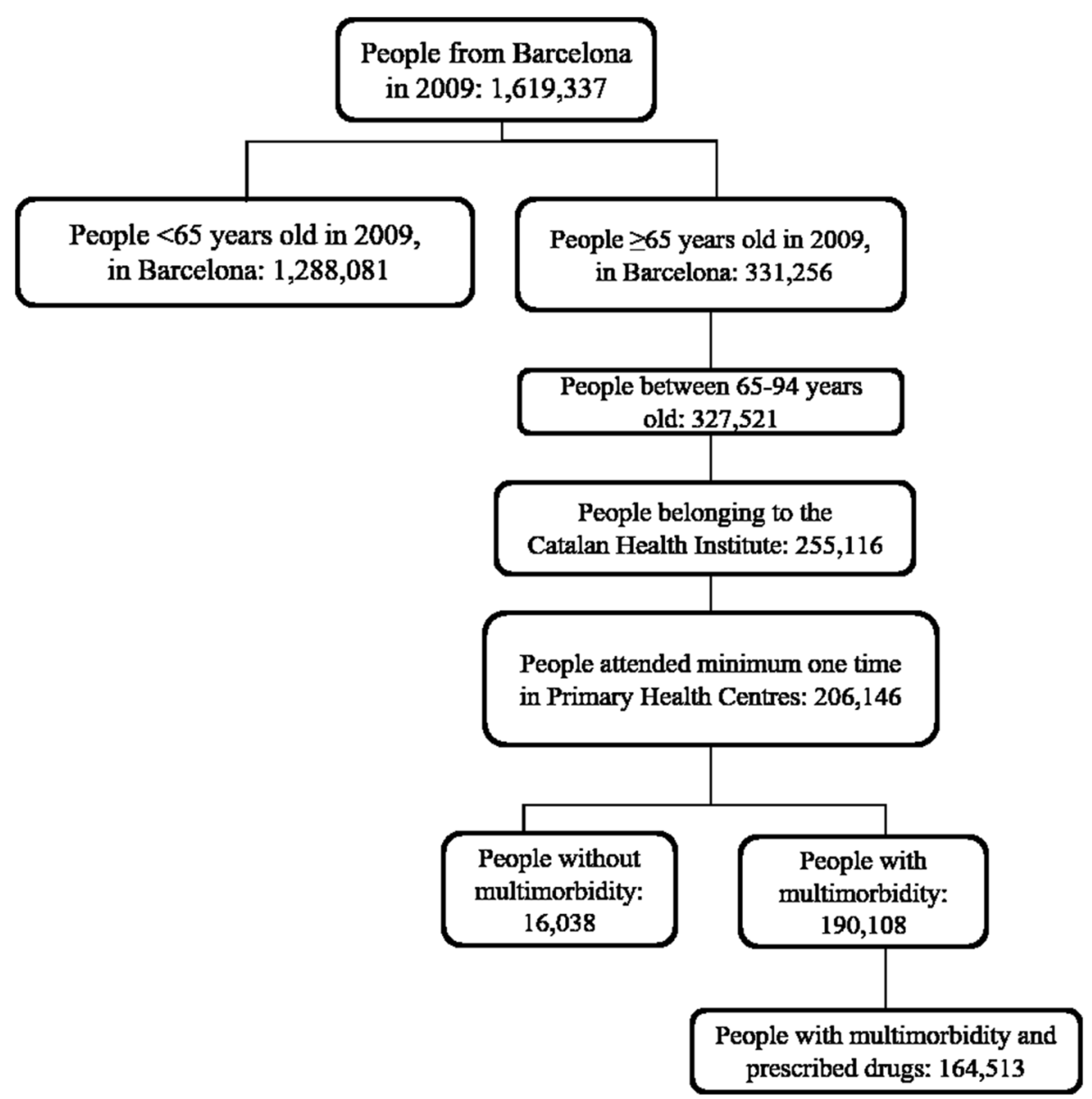

Fig. 1 Flow chart

means clustering. All analyses were stratified by sex and age.

\section{Multiple correspondence analysis}

MCA is a data analysis technique used to detect and represent underlying structures in sets of nominal categorical data. It identifies groups with similar characteristics and shows, in a multidimensional space, relationships between dichotomous or categorical variables (in our case drug prescriptions) that would be difficult to observe in a contingency table [30,31]. MCA also allows individuals to be directly represented as points (coordinates) in a geometric space through the transformation of original binary data to continuous ones. The MCA was based on the indicator matrix. The optimal number of dimensions extracted and percentages of inertia were determined by means of a scree plot.

\section{K-means clustering}

Using the geometric space created in the MCA, patients were classified into clusters according to proximity criteria by means of the k-means algorithm, and centers obtained for each cluster. The optimal number of clusters $(\mathrm{k})$, which is the solution with the highest CalinskiHarabaz index value, was assessed using criteria with 100 iterations. To assess internal cluster quality, cluster stability of the optimal solution was computed using Jaccard bootstrap values with 100 runs [17]. Highly stable clusters should yield average Jaccard similarities of 0.85 and above.

\section{Medication patterns}

To describe the medication patterns across the clusters, we used three criteria: a) the prevalence of prescribed drugs in each cluster; b) the observed/expected (O/E) ratios obtained by dividing the prevalence of a particular drug in each cluster by the prevalence of the same prescribed drug in the age and sex groups, considering over-represented drugs when value $\geq 2$; and c) exclusivity, defined as the proportion of individuals with a particular prescribed drug included in the cluster over the total number of individuals with a particular prescribed drug in the corresponding age and sex group, considering high exclusivity when value $\geq 50 \%$. 
Medication patterns were defined by considering drugs with a prevalence $\geq 20 \%$ or an $\mathrm{O} / \mathrm{E}$ ratio $\geq 2$. To identify the importance of each medication and, as a consequence, the amount of medication included in a cluster, we employed exclusivity. In order to facilitate the designation of a medication pattern we named the patterns considering medications belonging to the same ATC group with an exclusivity value $\geq 50 \%$, even when presenting a low prevalence. And we also took into consideration to name the pattern those drugs over-represented by $\mathrm{O} / \mathrm{E}$ ratio $\geq 2$. We then described medications included in each cluster using three numbers of characteristics: prevalent drugs (prevalence $\geq 20 \%$ ), drugs over-represented $(\mathrm{O} / \mathrm{E}$ ratio $\geq 2)$ and exclusive drugs (exclusivity $\geq 50 \%$ ). But we considered only exclusive and overrepresented drugs to label the pattern.

In addition to mathematical validation, clinical criteria based on previous literature [32-34] and clinical feedback from the research team ( 3 family physicians and 2 epidemiologists) were employed to evaluate the consistency and significance of the final cluster solution.

The analyses were carried out using SPSS for Windows, version 24 (SPSS Inc., Chicago, IL, USA) and R version 3.4.2 ( $\mathrm{R}$ Foundation for Statistical Computing, Vienna, Austria).

\section{Results}

The sample was composed of 164,513 patients aged $\geq 65$ years all of whom presented multimorbidity and had at least 1 drug prescribed; $66.8 \%$ were women. The group $65-79$ years had a mean age of 72.0 years $(\mathrm{SD}=4.3)$ and was prescribed a median of $4(\mathrm{IQR}=3-7)$ drugs. The group 80-94 years had a mean age of 84.1 years $(\mathrm{SD}=$ 3.4) and was prescribed a median of 6 (IQR: 4-8) drugs.
At least $45.9 \%$ of the $65-79$ year and $61.8 \%$ of the $80-$ 94 year groups were prescribed 5 or more drugs. As expected, the use of 10 or more drugs was almost twice in the 80-94 compared to the 65-79 year age group. The number of prescribed drugs and chronic diseases did not differ between sexes (Table 1). The 10 most widely prescribed drugs across the sample belonged to 3 ATC system groups: alimentary tract and metabolism (A), nervous system (N), and cardiovascular system (C). Proton pump inhibitors and HMG CoA reductase inhibitors were present in the top 3 most prescribed drugs in all groups, with platelet aggregation inhibitors (excluding heparin) in men and benzodiazepine derivatives (65-79 years) and anilides (80-94 years) for women (Table 2).

\section{Characteristics of medication patterns}

Six medication patterns for each age and sex group were identified. All the groups had a non-specific pattern consisting of highly prevalent drugs that were neither overrepresented nor exclusive. The other 5 patterns were made up of drugs belonging to 1 or more anatomical groups corresponding to: alimentary tract and metabolism (A), blood and blood forming organs (B), cardiovascular system (C), dermatological (D), musculoskeletal system $(\mathrm{M})$, nervous system $(\mathrm{N})$, respiratory system (R), and sensory organs (S) (Table 3, Additional files 2, 3 and 4).

As an example, findings for women $65-79$ years are represented in Table 3. Six medication patterns were identified, numbered according to the weight of the sample implied (descending order): non-specific (cluster 1), followed by nervous system (cluster 2), musculo-skeletal + dermatological (cluster 3), alimentary tract and metabolism (cluster 4), respiratory system (cluster 5), and cardiovascular system

Table 1 Descriptive data, by sex and age groups, of the multimorbid patients ( $n=164,513)$ aged $65-94$ years attended in 2009 at primary healthcare centres located in Barcelona

\begin{tabular}{|c|c|c|c|c|}
\hline & \multicolumn{2}{|l|}{ Women } & \multicolumn{2}{|l|}{ Men } \\
\hline & $65-79$ years & 80-94 years & $65-79$ years & 80-94 years \\
\hline Participants n (\%) & $78,008(47.4)$ & $31,848(19.4)$ & $41,931(25.5)$ & $12,726(7.7)$ \\
\hline \multicolumn{5}{|l|}{ Number drugs n (\%) } \\
\hline $1-4$ & $40,931(52.5)$ & $11,374(35.7)$ & $22,703(54.1)$ & $4868(38.3)$ \\
\hline $5-9$ & $31,500(40.4)$ & $16,460(51.7)$ & $16,339(39.0)$ & $6268(49.3)$ \\
\hline$\geq 10$ & $5577(7.1)$ & $4014(12.6)$ & $2889(6.9)$ & $1590(12.5)$ \\
\hline Median number of drugs $\left(\mathrm{IQR}^{\mathrm{a}}\right)$ & $4(3-7)$ & $6(4-8)$ & $4(2-6)$ & $5(3-8)$ \\
\hline \multicolumn{5}{|l|}{ Number of chronic diseases n (\%) } \\
\hline 2 & $2806(3.6)$ & $1125(3.5)$ & $1792(4.3)$ & $410(3.2)$ \\
\hline$[3-5]$ & $20,301(26.0)$ & $7689(24.1)$ & $12,484(29.8)$ & $3090(24.3)$ \\
\hline$[6-9]$ & 33,089 (42.4) & $13,495(42.4)$ & 17,955 (42.8) & $5562(43.7)$ \\
\hline$\geq 10$ & $21,812(28.0)$ & 9539 (30.0) & $9700(23.1)$ & 3664 (28.8) \\
\hline Median number of chronic diseases (IQR $\left.{ }^{\mathrm{a}}\right)$ & $7(5-10)$ & $8(5-10)$ & $7(5-9)$ & $7(5-10)$ \\
\hline
\end{tabular}


Table 2 The ten most commonly prescribed drugs in 2009 for multimorbid patients $(n=164,513)$ aged $65-94$ years, by sex and age groups, attended at primary healthcare centres located in Barcelona

\begin{tabular}{|c|c|c|c|c|c|c|c|c|}
\hline & \multicolumn{4}{|l|}{ Women } & \multicolumn{4}{|l|}{ Men } \\
\hline & $\overline{\text { ATC code }}{ }^{a}$ & Drug name & $\mathrm{N}$ & $\%$ & $\overline{\text { ATC } \text { code }^{a}}$ & Drug name & $\mathrm{N}$ & $\%$ \\
\hline \multirow[t]{10}{*}{$65-79$ years } & $\mathrm{A02BC}$ & Proton pump inhibitors & 32,634 & 41.8 & C10AA & HMG CoA reductase inhibitors & 18,188 & 43.4 \\
\hline & C10AA & HMG CoA reductase inhibitors & 32,004 & 41.0 & $\mathrm{~A} 02 \mathrm{BC}$ & Proton pump inhibitors & 15,170 & 36.2 \\
\hline & N05BA & Benzodiazepine derivatives & 20,649 & 26.5 & $\mathrm{~B} 01 \mathrm{AC}$ & Platelet aggregation inhibitors excl. Heparin & 13,872 & 33.1 \\
\hline & N02BE & Anilides & 18,434 & 23.6 & CO9AA & ACE inhibitors, plain & 9748 & 23.2 \\
\hline & $\mathrm{B} 01 \mathrm{AC}$ & $\begin{array}{l}\text { Platelet aggregation } \\
\text { inhibitors excl. Heparin }\end{array}$ & 15,338 & 19.7 & G04CA & Alpha-adrenoreceptor antagonists & 7235 & 17.3 \\
\hline & CO9AA & ACE inhibitors, plain & 13,578 & 17.4 & $\mathrm{~A} 10 \mathrm{BA}$ & Biguanides & 6306 & 15.0 \\
\hline & M05BA & Bisphosphonates & 13,309 & 17.1 & N05BA & Benzodiazepine derivatives & 6019 & 14.4 \\
\hline & N06AB & Selective serotonin reuptake inhibitors & 11,522 & 14.8 & C08CA & Dihydropyridine derivatives & 5996 & 14.3 \\
\hline & CO3AA & Thiazides, plain & 10,112 & 13.0 & CO7AB & $\begin{array}{l}\text { Beta blocking } \\
\text { agents, selective }\end{array}$ & 5934 & 14.2 \\
\hline & C09CA & Angiotensin II antagonists, plain & 9231 & 11.8 & $\mathrm{~N} 02 \mathrm{BE}$ & Anilides & 5625 & 13.4 \\
\hline \multirow[t]{10}{*}{ 80-94 years } & $\mathrm{A} 02 \mathrm{BC}$ & Proton pump inhibitors & 16,496 & 51.8 & $\mathrm{~A} 02 \mathrm{BC}$ & Proton pump inhibitors & 5877 & 46.2 \\
\hline & $\mathrm{N} 02 \mathrm{BE}$ & Anilides & 11,370 & 35.7 & $\mathrm{~B} 01 \mathrm{AC}$ & Platelet aggregation inhibitors excl. Heparin & 5641 & 44.3 \\
\hline & C10AA & HMG CoA reductase inhibitors & 11,222 & 35.2 & C10AA & HMG CoA reductase inhibitors & 4657 & 36.6 \\
\hline & B01AC & $\begin{array}{l}\text { Platelet aggregation } \\
\text { inhibitors excl. Heparin }\end{array}$ & 10,512 & 33.0 & CO9AA & ACE inhibitors, plain & 3235 & 25.4 \\
\hline & N05BA & Benzodiazepine derivatives & 9633 & 30.2 & N02BE & Anilides & 2638 & 20.7 \\
\hline & CO9AA & ACE inhibitors, plain & 7223 & 22.7 & G04CA & Alpha-adrenoreceptor antagonists & 2601 & 20.4 \\
\hline & C08CA & Dihydropyridine derivatives & 5283 & 16.6 & N05BA & Benzodiazepine derivatives & 2313 & 18.2 \\
\hline & C03CA & Sulfonamides, plain & 5265 & 16.5 & C08CA & Dihydropyridine derivatives & 2260 & 17.8 \\
\hline & N06AB & Selective serotonin reuptake inhibitors & 5258 & 16.5 & C03CA & Sulfonamides, plain & 1930 & 15.2 \\
\hline & CO9CA & Angiotensin II antagonists, plain & 4502 & 14.1 & C01DA & Organic nitrates & 1622 & 12.7 \\
\hline
\end{tabular}

Code $^{\mathrm{a}}$ : chemical subgroup, 4rt level, ATC code (Anatomical Therapeutic Chemical classification) from the World Health Organization (Additional file 1)

For more details, visit webside: https://www.whocc.no/atc/structure_and_principles/

(cluster 6). For each cluster, three subgroups of prescribed drugs that encompassed the pattern were defined. Three kinds of data were shown for every cluster. Using the example of the musculo-skeletal and dermatological pattern (cluster 3), we identified three different groups of drugs in the pattern:

a) drugs with a high prevalence but not over- represented such as proton pump inhibitors (prevalence 66\%, $\mathrm{O} / \mathrm{E}$ ratio 1.58 , exclusivity $19 \%$ ) and benzodiazepine derivatives (prevalence 33\%, O/E ratio 1.26, exclusivity $15 \%)$;

b) drugs with a high/low prevalence over-represented with exclusivity $<50 \%$ such as anilides (prevalence $61 \%$, $\mathrm{O} / \mathrm{E}$ ratio 2.57 , exclusivity $31 \%$ ) and other opioids (prevalence $10 \%, \mathrm{O} / \mathrm{E}$ ratio 3.25 , exclusivity $40 \%$ );

c) drugs with a high/low prevalence over-represented and with exclusivity $\geq 50 \%$ such as anti-inflammatory preparations, non-steroids for topical use (prevalence $33 \%$, O/E ratio 5.96, exclusivity $70 \%$ ) and potent corticosteroids (group III) (prevalence 9\%, O/E ratio 6.65, exclusivity $81 \%$ ) (Table 3 ).
It was observed that the non-specific pattern had the greatest number of patients for all groups and was defined by drugs that were neither prevalent nor overrepresented. With respect to the non-specific pattern, the number of patients aged 65-79 years was higher than those aged 80-94 years for both sexes. According to the frequency of patients, the next patterns were: for women 65-79 years "nervous system" and "musculo-skeletal + dermatological", whilst for women 80-94 years they included alimentary tract and metabolism as a drug group implied in frequency; for men 65-79 years they were "cardiovascular system" and "alimentary tract and metabolism", and for those 80-94 years was added the drug group related to musculo-skeletal and nervous system (Table 3, Additional files 2, 3 and 4).

Comparing patterns between sexes, women had four patterns in both age groups which implied only one over-represented anatomical system (alimentary tract and metabolism, cardiovascular system, nervous system, and respiratory system), in contrast to men who had only two patterns implying one anatomical 
Table 3 Example of medication patterns across women 65-79 years attended in primary health centres in Barcelona during 2009 $(N=78,008)$

Code $^{\&}$ Drugs $\operatorname{Pre}^{\mathrm{b}} \underset{\text { ratio }^{a}}{\mathrm{O} / \mathrm{E}}$ Exclus.

Cluster $1 n=39,202(50 \%)$

Non-specific pattern

C10AA HMG CoA reductase inhibitors

A02BC Proton pump inhibitors

Cluster $2 n=14,604$ (19\%)

Nervous system pattern

Cluster $3 n=9502$ (12\%)

"Musculo-skeletal system" and

"Dermatologicals" pattern

A02BC Proton pump inhibitors

$\begin{array}{ll}\text { A02BC } & \text { Proton pump inhibitors } \\ \text { N05BA } & \text { Benzodiazepine derivatives } \\ \text { C10AA } & \text { HMG CoA reductase inhibitors } \\ \text { N06AB } & \text { Selective serotonin reuptake inhibitors } \\ \text { B01AC Platelet aggregation inhibitors excl. Heparin } \\ \text { M05BA Bisphosphonates } \\ \text { N02BE Anilides } \\ \text { N06AX Other antidepressants } \\ \text { N03AX Other antiepileptics } \\ \text { N05CD Benzodiazepine derivatives } \\ \text { A12AA Calcium } \\ \text { N06AA Non-selective monoamine reuptake inhibitors } \\ \text { C01DA Organic nitrates } \\ \text { N02AX Other opioids } \\ \text { A11CC Vitamin D and analogues } \\ \text { A06AD Osmotically acting laxatives } \\ \text { N03AE } \quad \text { Benzodiazepine derivatives } \\ \text { C07AA Beta blocking agents, non-selective } \\ \text { H02AB Glucocorticoids } \\ \text { C10AX Other lipid modifying agents } \\ \text { N06DX Other anti-dementia drugs } \\ \text { A03FA Propulsive }\end{array}$

N02BE Anilides

N05BA Benzodiazepine derivatives

M02AA Antiinflammatory preparations, non-steroids for tropical use

C10AA HMG CoA reductase inhibitors

M01AE Propionic acid derivatives

C05CA Bioflavonoids

M01AX Other antiinflammatory and antirheumatic agents, non-steroids

A02AD Combinations and complexes of aluminium, calcium and magnesium compounds

M01AB Acetic acid derivatives and related substances

D01AC Imidazole and triazole derivatives

N02AX Other opioids

D07AC Corticosteroidas, potent (group III)

N02BB Pyrazolones

\begin{tabular}{lll}
$70 \%$ & 1.68 & $31 \%$ \\
$58 \%$ & 2.18 & $41 \%$ \\
$56 \%$ & 1.36 & $25 \%$ \\
$40 \%$ & 2.71 & $51 \%$ \\
$35 \%$ & 1.76 & $33 \%$ \\
$28 \%$ & 1.62 & $30 \%$ \\
$26 \%$ & 1.10 & $21 \%$ \\
$14 \%$ & 3.49 & $65 \%$ \\
$10 \%$ & 3.16 & $59 \%$ \\
$9 \%$ & 2.03 & $38 \%$ \\
$7 \%$ & 2.58 & $48 \%$ \\
$7 \%$ & 3.07 & $57 \%$ \\
$7 \%$ & 2.32 & $43 \%$ \\
$6 \%$ & 2.03 & $38 \%$ \\
$6 \%$ & 2.97 & $56 \%$ \\
$6 \%$ & 2.04 & $38 \%$ \\
$4 \%$ & 3.69 & $69 \%$ \\
$4 \%$ & 2.61 & $49 \%$ \\
$4 \%$ & 2.71 & $51 \%$ \\
$4 \%$ & 2.25 & $42 \%$ \\
$3 \%$ & 2.44 & $46 \%$ \\
$3 \%$ & 2.25 & $42 \%$ \\
\hline
\end{tabular}

$66 \% \quad 1.58 \quad 19 \%$

$15 \% \quad 5.17 \quad 63 \%$

$32 \% \quad 0.78 \quad 39 \%$

$21 \% \quad 0.51 \quad 26 \%$

$\begin{array}{lll}61 \% & 2.57 \quad 31 \%\end{array}$

$33 \% \quad 1.26 \quad 15 \%$

$31 \% \quad 5.96 \quad 73 \%$

$30 \% \quad 0.74 \quad 9 \%$

$27 \% \quad 4.30 \quad 52 \%$

$19 \% \quad 2.88 \quad 35 \%$

$\begin{array}{lll}17 \% & 2.84 \quad 35 \%\end{array}$

$15 \% \quad 4.24 \quad 52 \%$

10\% $5.93 \quad 72 \%$

$10 \% \quad 3.25 \quad 40 \%$

$9 \% \quad 6.65 \quad 81 \%$

$6 \% \quad 5.30 \quad 65 \%$ 
Table 3 Example of medication patterns across women 65-79 years attended in primary health centres in Barcelona during 2009 $(N=78,008)$ (Continued)

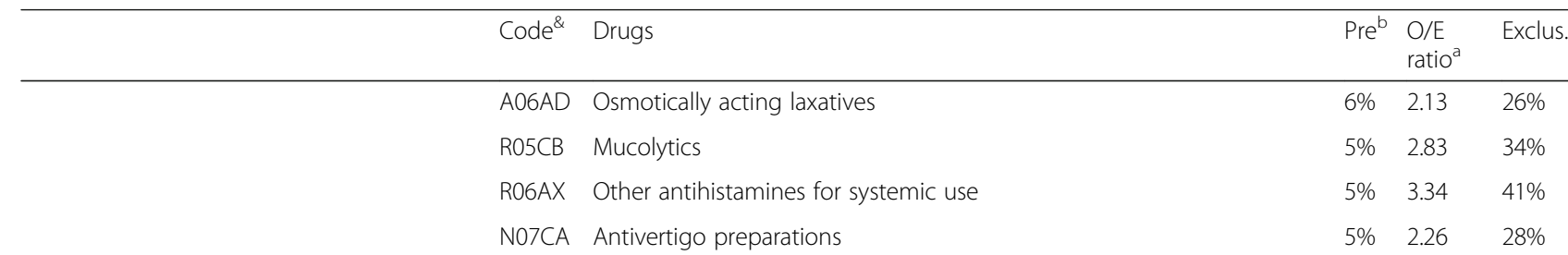

Cluster $4 n=8745(11 \%)$

Alimentary tract and metabolism pattern
C10AA HMG COA reductase inhibitors

A10BA Biguanides

B01AC Platelet aggregation inhibitors excl. Heparin

A02BC Proton pump inhibitors

A10BB Sulfonylureas

C09AA ACE inhibitors, plain

C08CA Dihydropyridine derivatives

C07AB Beta blocking agents, selective

N02BE Anilides

A10AC Insulins and analogues for injection, intermediate-acting

C01DA Organic nitrates

A10AE Insulins and analogues for injection, long-acting

M04AA Preparations inhibiting uric acid production

C02CA Alpha-adrenoreceptor antagonists

C10AB Fibrates

A10AD Insulins and analogues for injection, intermediate- or long- acting combined with fast- acting

G04CA Alpha-adrenoreceptor antagonists

C10AX Other lipid modifying agents

\begin{tabular}{lll}
$68 \%$ & 1.67 & $19 \%$ \\
$65 \%$ & 5.86 & $66 \%$ \\
$61 \%$ & 3.12 & $35 \%$ \\
$50 \%$ & 1.19 & $13 \%$ \\
$37 \%$ & 6.69 & $75 \%$ \\
$30 \%$ & 1.70 & $19 \%$ \\
$29 \%$ & 2.68 & $30 \%$ \\
$23 \%$ & 2.18 & $24 \%$ \\
$22 \%$ & 0.94 & $10 \%$ \\
$10 \%$ & 6.34 & $71 \%$ \\
\hline $9 \%$ & 3.22 & $36 \%$ \\
$9 \%$ & 6.04 & $68 \%$ \\
\hline $9 \%$ & 2.97 & $33 \%$ \\
$7 \%$ & 3.57 & $40 \%$ \\
$7 \%$ & 2.96 & $33 \%$ \\
$7 \%$ & 6.24 & $70 \%$ \\
\hline $4 \%$ & 2.13 & $24 \%$ \\
\hline $4 \%$ & 2.36 & $26 \%$
\end{tabular}

Cluster $5 n=3275$ (4\%)

Respiratory system pattern

\author{
R03AC Selective beta-2-adrenoreceptor agonists \\ A02BC Proton pump inhibitors \\ R03BB Anticholinergics \\ R03AK Adrenergics in combination with corticosteroids or other drugs, excl. \\ Anticholinergics \\ R03BA Glucocorticoids \\ C10AA HMG COA reductase inhibitors \\ N02BE Anilides \\ N05BA Benzodiazepine derivatives \\ B01AC Platelet aggregation inhibitors excl. Heparin \\ C03CA Sulfonamides, plain \\ R05CB Mucolytics \\ R06AX Other antihistamines for systemic use \\ C08DB Benzothiazepine derivatives \\ H02AB Glucocorticoids \\ G04CA Alpha-adrenoreceptor antagonists
}

$\begin{array}{lll}72 \% & 16.88 & 71 \% \\ 54 \% & 1.30 & 5 \% \\ 54 \% & 18.86 & 79 \% \\ 51 \% & 11.87 & 50 \% \\ 40 \% & 18.45 & 77 \% \\ 38 \% & 0.94 & 4 \% \\ 32 \% & 1.36 & 6 \% \\ 27 \% & 1.01 & 4 \% \\ 23 \% & 1.17 & 5 \% \\ 14 \% & 2.11 & 9 \% \\ 11 \% & 6.34 & 27 \% \\ 6 \% & 3.79 & 16 \% \\ 5 \% & 2.26 & 9 \% \\ 4 \% & 3.07 & 13 \% \\ 4 \% & 2.02 & 8 \%\end{array}$


Table 3 Example of medication patterns across women 65-79 years attended in primary health centres in Barcelona during 2009 $(N=78,008)$ (Continued)

Code $^{\&}$ Drugs Pre $^{\mathrm{b}} \underset{\text { ratio }^{\mathrm{a}}}{\mathrm{O} / \mathrm{a}}$ Exclus.

Cluster $6 n=2680(3 \%)$

Cardiovascular system pattern

B01AA Vitamin K antagonists
C03CA Sulfonamides, plain
A02BC Proton pump inhibitors
C01AA Digitalis glycosides
C10AA HMG CoA reductase inhibitors
N02BE Anilides
N05BA Benzodiazepine derivatives
C09AA ACE inhibitors, plain
C07AB Beta blocking agents, selective
C09CA Angiotensin II antagonists, plain
A12BA Potassium
C03DA Aldosterone antagonists
C08DB Benzothiazepine derivatives
C07AG Alpha and beta blocking agents
M04AA Preparations inhibiting uric acid production
C01DA Organic nitrates
B03AA Iron bivalent, oral preparations
A10AE Insulins and analogues for injection, long-acting
A02BA H2 - receptor antagonists

\begin{tabular}{rrr}
$78 \%$ & 15.74 & $54 \%$ \\
$66 \%$ & 10.05 & $35 \%$ \\
$58 \%$ & 1.37 & $5 \%$ \\
$53 \%$ & 27.60 & $95 \%$ \\
$43 \%$ & 1.06 & $4 \%$ \\
$32 \%$ & 1.35 & $5 \%$ \\
$29 \%$ & 1.11 & $4 \%$ \\
$27 \%$ & 1.56 & $5 \%$ \\
$24 \%$ & 2.25 & $8 \%$ \\
\hline $23 \%$ & 1.98 & $7 \%$ \\
\hline $20 \%$ & 16.35 & $56 \%$ \\
$19 \%$ & 17.02 & $58 \%$ \\
$13 \%$ & 6.07 & $21 \%$ \\
$12 \%$ & 8.91 & $31 \%$ \\
\hline $11 \%$ & 3.73 & $13 \%$ \\
\hline $9 \%$ & 3.26 & $11 \%$ \\
$5 \%$ & 3.60 & $12 \%$ \\
$3 \%$ & 2.30 & $8 \%$ \\
$3 \%$ & 2.12 & $7 \%$ \\
\hline
\end{tabular}

Code $^{\&}$ :Chemical subgroup, 4rt level, ATC code (Anatomical Therapeutic Chemical classification) from the World Health Organization O/E ratio ${ }^{a}$ : Observed/expected ratio

Pre ${ }^{\mathrm{b}}$ : Prevalence

Exclus.: Exclusivity

Selected criteria: Prevalence $\geq 20$ or Observed/Expected ratio $\geq 2$

system (cardiovascular and respiratory system). The other patterns were formed by two or more anatomical systems. The rest of the results are detailed in Table 3 and Additional files 2, 3 and 4.

Comparing patterns between age groups, no significant differences were observed for women with the exception of additional drugs encompassing the non-specific pattern (anilides, ACE inhibitors, benzodiazepine derivatives) (Table 3 , Additional file 2). The men's patterns, however, appeared more complex: to the non-specific pattern were added two drugs (platelet aggregation inhibitors excluding heparin and proton pump inhibitors), and in the 80-94 age group the patterns encompassed multiple anatomical groups including a sensory organs pattern (Additional files 3 and 4).

\section{Discussion}

In this study, we present data regarding prescription drugs in an urban population of elderly adults with multimorbidity. Prescription rates were high, particularly in the older subset of patients, probably due to the greater burden of chronic disease. Proton pump inhibitors were the most widely prescribed drug with cardiovascular and neurological drugs representing the most frequently prescribed groups. We defined 6 medication patterns which provide information about the multiple drugs grouped closely together in elderly patients. The pattern with the most participants, non-specific, had up to $39 \%$ of the age-sex sample included and was composed of drugs corresponding to specific diseases (hypertension, lipid disorder, depressive disorder (women)) and others related to the secondary prevention of cardiovascular/digestive diseases (platelet aggregation inhibitors and proton pump inhibitors). The rest of the medication patterns could be linked to the multimorbidity ones defined in a previous article performed in the same sample [35].

\section{Comparison with published literature}

Ageing is associated with functional decline, and the prescription of multiple drugs tends to be highest in the oldest segments of the population [36]. Just over half the patients in our study had been prescribed 5 or more drugs, rates of between 45.0 and $80.0 \%$ have been previously described based on primary care EHR [9, 37]. These results showed that the 10 most prescribed drugs 
were to treat metabolic, cardiovascular, and nervous system disorders, in line with other reports for the elderly $[35,38,39]$. As expected, considering that heart disease is the leading cause of death in such populations [40], cardiovascular drugs were the main group of prescribed drugs. Looking more closely, proton pump inhibitors were the most widely prescribed drug in our study, contrasting with findings on the prevalence of digestive tract chronic diseases conducted in the same sample [35]. Off-label use of proton pump inhibitors could be related to the prevention of adverse gastrointestinal effects, as reported elsewhere [41]. In addition, a high prevalence of lipid modifying (C10AA) agents and antithrombotic drugs (B01AC) was probably linked to their use in the primary and secondary prevention of thrombotic events. We would like to point out that benzodiazepines, despite their potentially adverse effects for older adults (e.g, memory impairment, delirium, falls) [42, 43], were still frequently prescribed in our population (from 14.4\% in men $65-79$ years to $30.2 \%$ in women $80-94$ years), with a reported prevalence among the elderly from 10.0 to $41.6 \%[44,45]$.

Six patterns per group defining user profiles with prescribed drugs were obtained. We took into account prescribed drugs, instead of consumed ones, because we assumed patients followed what their doctors suggested. As we studied patients with multimorbidity, we considered chronic drugs rather than supplements or acute prescriptions. As a result, many of the defined patterns seemed logical and in concordance with chronic disease prevalence [35]. In addition, differences in intra- and inter-patterns were represented defining prevalence, $\mathrm{O} / \mathrm{E}$ ratio, and exclusivity for each drug. The relevance of the prescribed drug was thus represented by these three parameters.

The non-specific pattern had the greatest number of patients in all strata as no anatomical group was overrepresented. It could, therefore, be hypothesized that patients evolve to 5 specific patterns across time, that is to say, the non-specific pattern could represent a pre-state of a specific one. In addition, the fact that the number of patients included in the non-specific pattern was lower in the 80-94 than the 65-79 year group points to the hypothesis that this pattern could be a pre-specific medication one. Nevertheless, longitudinal analyses should be conducted to substantiate this issue. With respect to specific patterns, the men's appeared more complex than women's possibly because of the anatomical systems involved and male smoking habits [46]. In concordance with this difference, more men in the 65-79 year group presented cardiovascular and respiratory patterns than women who showed mostly neuromuscular drug-related patterns. Furthermore, the fact that the patterns of the older participants were made up of more than one anatomical system was possibly related to the burden of chronic disease associated with age [23]. The observed medication patterns should coincide with the multimorbidity ones given that the former reflect the various illnesses being treated. For instance, if we compare multimorbidity and medication patterns from the same sample, the endocrine-metabolic multimorbidity pattern should be related to the alimentary tract and metabolism one [35]. A concept that concurs with a number of publications that have reported that medication data may represent a way of identifying chronic conditions [47]. Following this idea, medication patterns could help characterise individuals with multimorbidity. Finally, the use of three criteria to define patterns permitted a representation of all drugs, including those related to low prevalence diseases. Variability between chronic diseases and treatments was thus respected in our results.

To the best of our knowledge, only one study has previously defined medication patterns using EFA [22], and few authors have investigated such patterns in patients with multimorbidity [16]. It is difficult to draw comparisons because of differences in drug inclusion criteria, number of drugs considered, and especially methodology. Nevertheless, some anatomical systems, including cardiovascular, respiratory, and neurological ones were the same. Such similarities are probably related to the strong prevalence of chronic conditions. Nevertheless, with CA we obtained 6 markedly different patterns, and with the $\mathrm{O} / \mathrm{E}$ ratio and the exclusivity criteria we could define which drugs were over-represented, playing a more crucial role.

A recent publication has established that guidelines addressing polymedication appear arbitrary [15]. Our research thus contributes to the definition of medication patterns which could be used to identify both user profiles and safety issues (e.g. detecting prescription errors, for instance inappropriate drugs, or drug-drug associations), something that is not possible with multimorbidity patterns. The definition of medication patterns could open new paths to create instruments to prioritize groups of individuals and permit effective prescription. In addition, establishing medication patterns in accordance to multimorbidity patterns would help to determine prognostic factors in drug safety, define possible adverse drug reactions, and identify drug-drug and drugdisease interactions. The analysis of medication patterns thus provides an additional perspective for interpreting and defining the population's health.

\section{Strength and weakness}

Our study sample is both reliable and representative of the population, thus adding robustness to our results. Moreover, we provide an accurate reflection of real prescribing habits for the elderly with multimorbidity in an urban public 
primary health care setting. Analyses of individual medication patterns can lead to new insights into individual prescription situations. We consider that complexity among patients is well represented in these patterns. However, some limitations should be considered. On one hand, selected criteria of chronicity (prescription of 6 or more months) may have caused a selection bias, although we followed an established definition [23]. In addition, we have to assume that $\mathrm{CA}$ is inherently exploratory in nature and different clustering algorithms may produce varying results. The lack of studies defining medication patterns also limits comparisons between results and populations. Finally, we should consider as a limitation the fact that the collected data were 10 years old and may not exactly reflect current prescription patterns. Nevertheless, these medication patterns correspond to a sixyear longitudinal multimorbidity study $[35,48]$ in which it was observed that multimorbidity patterns did not differ at all during the period studied. In addition, in public primary health care, the implementation of new treatments for specific diseases (for example, oral anticoagulants or oral antidiabetic medications) are not yet generalised. For this reason, we considered that the medication patterns represented current prescription.

\section{Future research}

Medication patterns could change with time as a consequence of multimorbidity evolution and new treatments applied in some chronic diseases. Our study is crosssectional, but in future research it would be advantageous to analyse large prospective cohorts with different estimates to define medication patterns and identify their stability or evolution. In addition, generational differences are expected due to modified lifestyle habits. Thus, reanalyses should be considered as medication patterns are expected to alter across decades.

Taking into account drug prescription and medication patterns, improvements in guidelines for the clinical management of elderly patients should be contemplated. In addition, the methodology used for clustering could be a starting point for analysing drug safety in relation to drug interaction.

\section{Conclusions}

This study provides information about prescription drugs in an urban population of older adults with multimorbidity. Our results showed highly elevated prescription rates, particularly in the older subset of patients, probably due to the greater burden of chronic disease. Clinical practice should consider reviewing off-label prescribed drugs for possible de-prescription.

The study of medication patterns provides a method for analysing the use of multiple drugs in elderly patients. We identified 6 medication patterns in our series which could provide new avenues for evaluating multimorbidity.

\section{Additional files}

Additional file 1: The main groups of the Anatomical Therapeutic Chemical (ATC) system. (DOCX $12 \mathrm{~kb}$ )

Additional file 2: Medication patterns across women 80-94 years attended in primary health centres in Barcelona during $2009(N=31,848)$. Selected criteria: Prevalence $\geq 20$ or Observed/Expected ratio $\geq 2$. (DOCX $24 \mathrm{~kb})$

Additional file 3: Medication patterns across men 65-79 years attended in primary health centres in Barcelona during $2009(N=41,931)$. Selected criteria: Prevalence $\geq 20$ or Observed/Expected ratio $\geq 2$. (DOCX 26 kb)

Additional file 4: Medication patterns across men 80-94 years attended in primary health centres in Barcelona during $2009(N=12,726)$. Selected criteria: Prevalence $\geq 20$ or Observed/Expected ratio $\geq 2$. (DOCX 23 kb)

\section{Abbreviations}

ATC: Anatomical Therapeutic Chemical; CA: Cluster analysis; EFA: Exploratory factor analysis; EHR: Electronic health records; Exclus: Exclusivity; IDIAPJGol: Institut Universitari d'Investigació en Atenció Primària Jordi Gol; IQR: Interquartile range; MCA: Multiple correspondence analysis; O/E ratios: Observed/expected ratios; PHCC: Primary health care centres; Pre: Prevalence; SD: Standard deviation; SIDIAP: System for Research in Primary Care

\section{Acknowledgements}

We thank the Catalan Health Institute, and especially the Information System for Research in Primary Care (SIDIAP) Unit which provided the study database.

\section{Authors' contributions}

All authors contributed to the design of the study, revised the article, and approved the final version. MGC, CV, QFB, ARL, TLJ, MAM, MPV, drafted the study protocol and obtained the funding. MGC, CV, ARL, TLJ contributed to the analysis and interpretation of data. MGC, CV, QFB, TLJ; ARL, MAM, MPV wrote the first draft, and all authors contributed ideas, interpreted the findings and reviewed rough drafts of the manuscript. All the authors read and approved the final manuscript.

\section{Funding}

This manuscript is part of a PhD being undertaken by MGC at the Public Health Department (Departament de Pediatria, d'Obstetrícia i Ginecologia i de Medicina Preventiva) at the Universitat Autònoma de Barcelona. This work was supported by a pre-doctoral grant from Catalan Health Institute in Barcelona; by the Catalan Society of General Practitioners (CAMFiC) and by SIDIAP grant to MGC in 2015; this latter organization allowed us to explore their dataset to obtain the results. The funders had no role in the study design or data collection, analysis, and interpretation, writing of the manuscript, and decision to submit for publication.

\section{Availability of data and materials}

The data that support the findings of this study may be obtained from SIDIAP but restrictions could apply to those used under license. Upon reasonable request and with permission of SIDIAP they may be available from the authors.

\section{Ethics approval and consent to participate}

The protocol of the study was approved by the Committee of the Ethics of Clinical Research, Institut Universitari d'Investigació en Atenció Primària Jordi Gol (IDIAP Jordi Gol) (Protocol No: P15/149). All data were anonymized, and the confidentiality of EHR was respected at all times in accordance with national and international law. As all data were anonymized, no consent to individuals were required.

Consent for publication

Not applicable.

Competing interests

The authors declare that they have no competing interests. 


\section{Author details}

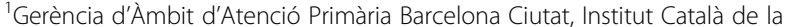
Salut, Carrer Balmes 22, Barcelona, Spain. ${ }^{2}$ Institut Universitari d'Investigació en Atenció Primària Jordi Gol (IDIAP Jordi Gol), Gran Via Corts Catalanes, 587 àtic, 08007 Barcelona, Spain. ${ }^{3}$ Universitat Autònoma de Barcelona, Plaça Cívica, 08193 Bellaterra, Barcelona, Spain. ${ }^{4}$ Facultat d'infermeria, Universitat de Girona, Emili Grahit, 77, 17071 Girona, Spain. ${ }^{5}$ Unitat de Suport a la Recerca de Barcelona, Institut Universitari d'Investigació en Atenció Primària Jordi Gol (IDIAP Jordi Gol), Carrer Sardenya, 375, 08025 Barcelona, Spain. ${ }^{6}$ Departament de psiquiatria, Hospital Universitari de Vic, Francesc Pla el Vigatà, 1, 08500 Vic, Barcelona, Spain. 'Facultat de Ciències de la Salut i Benestar, Universitat de Vic - Universitat Central de Catalunya, Sagrada Família, 7, 08500 Vic, Spain.

\section{Received: 30 October 2018 Accepted: 23 May 2019}

Published online: 13 June 2019

\section{References}

1. Murray CJL, Barber RM, Foreman KJ, Ozgoren AA, Abd-Allah F, Abera SF, et al. Global, regional, and national disability-adjusted life years (DALYs) for 306 diseases and injuries and healthy life expectancy (HALE) for 188 countries, 1990-2013: quantifying the epidemiological transition. Lancet. 2015:19902013.

2. Oeppen J, Vaupel JW. Demography. Broken Limits to Life Expectancy. Science (80- ). 2002;296(May):1029-31.

3. Eurostat. Population structure and ageing - statistics explained. 2016. http:// ec.europa.eu/eurostat/statistics-explained/index.php/Population_structure_ and_ageing. Accessed 23 Aug 2017.

4. Eurostat. Active ageing - employment, social affairs \& inclusion - European Commission. 2015. http://ec.europa.eu/social/main.jsp?catld=1062. Accessed 23 Aug 2017.

5. Jacob L, Breuer J, Kostev K. Prevalence of chronic diseases among older patients in German general practices. GMS Ger Med Sci. 2016;14:1-7.

6. Marengoni A, Angleman S, Melis R, Mangialasche F, Karp A, Garmen A, et al. Aging with multimorbidity: a systematic review of the literature. Ageing Res Rev. 2011 Mar 23;10(4):430-9.

7. Van den AM, Buntinx F. Multimorbidity in general practice: prevalence, incidence, and determinants of co-occurring chronic and recurrent diseases. J Clin Epidemiol. 1998;51(5):367-75.

8. Wehling M. Multimorbidity and polypharmacy: how to reduce the harmful drug load and yet add needed drugs in the elderly? Proposal of a new drug classification: fit for the aged: letters to the editor. J Am Geriatr Soc. 2009 Mar;57(3):560-1.

9. Freund J, Meiman J, Kraus C. Using electronic medical record data to characterize the level of medication use by age-groups in a network of primary care clinics. J Prim Care Community Heal. 2013;4(4):286-93.

10. Mangoni AA, Jackson SHD. Age-related changes in pharmacokinetics and pharmacodynamics: basic principles and practical applications. Br J Clin Pharmacol. 2003:57(1):6-14.

11. Hajjar ER, Cafiero AC, Hanlon JT. Polypharmacy in elderly patients. Am J Geriatr Pharmacother. 2007 Dec;5(4):345-51.

12. Salazar JA, Poon I, Nair M. Clinical consequences of polypharmacy in elderly: expect the unexpected, think the unthinkable. Expert Opin Drug Saf. 2007 Nov 30;6(6):695-704

13. Gómez C, Vega-Quiroga S, Bermejo-Pareja F, Medrano MJ, Louis ED, BenitoLeón J. Polypharmacy in the elderly: a marker of increased risk of mortality in a population-based prospective study (NEDICES). Gerontology. 2015;61: 301-9.

14. Oscanoa TJ, Lizaraso F, Carvajal A. Hospital admissions due to adverse drug reactions in the elderly. A meta-analysis. Eur J Clin Pharmacol. 2017 Jun 1; 73(6):759-70.

15. Muth C, Blom JW, Smith SM, Johnell K, Gonzalez-Gonzalez Al, Nguyen TS, et al. Evidence supporting the best clinical management of patients with multimorbidity and polypharmacy: a systematic guideline review and expert consensus. J Intern Med. 2019;285(3):272-88.

16. Doos L, Roberts EO, Corp N, Kadam UT. Multi-drug therapy in chronic condition multimorbidity: a systematic review. Fam Pract. 2014;31(6):654-63.

17. Everitt BS, Landau S, Leese M, Stahl D. Cluster analysis. 5th ed: In John Wiley \& Sons, Ltd; 2011. p. 321-30.

18. Roso-Llorach A, Violán C, Foguet-Boreu Q, Rodriguez-Blanco T, Pons-Viqués M, Pujol-Ribera E, et al. Comparative analysis of methods for identifying multimorbidity patterns: a study of 'real-world' data. BMJ Open. 2018;8(3): e018986.

19. Britt HC, Harrison CM, Miller GC, Knox SA. Prevalence and patterns of multimorbidity in Australia. Med J Aust. 2008;189(2):72-7.

20. Steinman MA, Lee SJ, John Boscardin W, Miao Y, Fung KZ, Moore KL, et al. Patterns of multimorbidity in Eldery veterans. J Am Geriatr Soc. 2012;29(10):997-1003.

21. Foguet-Boreu Q, Violán C, Rodriguez-Blanco T, Roso-Llorach A, Pons-Vigués M, Pujol-Ribera E, et al. Multimorbidity patterns in elderly primary health care patients in a South Mediterranean European region: a cluster analysis. PLoS One. 2015;10(11):1-14.

22. Calderón-Larrañaga A, Gimeno-Feliu LA, González-Rubio F, Poblador-Plou B, Lairlasan José M, Abad-Díez JM, et al. Polypharmacy patterns: unravelling systematic associations between prescribed medications. PLoS One. 2013;8(12):1-10.

23. Violan C, Foguet-Boreu Q, Flores-Mateo G, Salisbury C, Blom J, Freitag M, et al. Prevalence, determinants and patterns of multimorbidity in primary care: a systematic review of observational studies. PLoS One. 2014;9(7):3-11.

24. Prados-Torres A, Calderón-Larrañaga A, Hancco-Saavedra J, Poblador-Plou B, Van Den Akker M. Multimorbidity patterns: a systematic review. J Clin Epidemiol. 2014:67(3):254-66.

25. García-Gil MDM, Hermosilla E, Prieto-Alhambra D, Fina F, Rosell M, Ramos R, et al. Construction and validation of a scoring system for the selection of high-quality data in a Spanish population primary care database (SIDIAP). Inform Prim Care. 2011 Jan;19(3):135-45.

26. Sistema d'informació per al desenvolupament de la Investigació en Atenció Primària.Inicio. http://www.sidiap.org/index.php/es. Accessed 6 Feb 2018.

27. Valderas JM, Starfield B, Sibbald B, Salisbury C, Roland M. Defining comorbidity: implications for understanding health and health services. Ann Fam Med. 2009 Jan;7(4):357-63.

28. Christian Berg Ms, Hege Salvesen Blix Ms, Irene Litleskare Ms, Marit Rønning Ms, Solveig Sakshaug Ms, Hanne Strøm Ms, et al. Guidelines for ATC classification and DDD assignment 2013. 16th editi. Oslo; 2013. 1-284 p. https://www.whocc.no/filearchive/publications/1_2013guidelines.pdf. Accessed 25 Jul 2017.

29. O'Halloran J, Miller GC, Britt H. Defining chronic conditions for primary care with ICPC-2. Fam Pract. 2004 Aug;21(4):381-6.

30. Sourial N, Wolfson C, Zhu B, Quail J, Fletcher J, Karunananthan S, et al. Correspondence analysis is a useful tool to uncover the relationships among categorical variables. J Clin Epidemiol. 2010 Jun;63(6):638-46.

31. García-Gil M, Blanch J, Comas-Cufí M, Daunis-i-Estadella J, Bolíbar B, Martí R, et al. Patterns of statin use and cholesterol goal attainment in a high-risk cardiovascular population: a retrospective study of primary care electronic medical records. J Clin Lipidol. 2016 Jan;10(1):134-42.

32. Evidence-based clinical decision support at the point of care | UpToDate. https://www.uptodate.com/home. Accessed 23 Jul 2017.

33. Cochrane Library. https://www.cochranelibrary.com/search. Accessed 21 Jul 2017.

34. Guies de pràctica clínica. Institut Català de la Salut. http://ics.gencat.cat/ca/ assistencia/coneixement-assistencial/guies-de-practica-clinica/. Accessed 10 Sept 2017.

35. Guisado-Clavero M, Roso-Llorach A, López-Jimenez T, Pons-Vigués M, Foguet-Boreu Q, Muñoz MA, et al. Multimorbidity patterns in the elderly: a prospective cohort study with cluster analysis. BMC Geriatr. 2018:18(1):16.

36. Payne RA, Avery AJ, Duerden M, Saunders CL, Simpson CR, Abel GA. Prevalence of polypharmacy in a Scottish primary care population. Eur J Clin Pharmacol. 2014;70(5):575-81.

37. Buck MD, Atreja A, Brunker CP, Jain A, Suh TT, Palmer RM, et al. Potentially inappropriate medication prescribing in outpatient practices: prevalence and patient characteristics based on electronic health records. Am J Geriatr Pharmacother. 2009:7(2):84-92.

38. Slabaugh SL, Maio V, Templin M, Abouzaid S. Prevalence and risk of polypharmacy among the elderly in an outpatient setting. Drugs Aging. 2010 Dec;27(12):1019-28.

39. Mizokami F, Koide $Y$, Noro T, Furuta K. Polypharmacy with common diseases in hospitalized elderly patients. Am J Geriatr Pharmacother. 2012 Apr;10(2):123-8.

40. Jackson CF, Wenger NK. Cardiovascular disease in the elderly. Rev Española Cardiol (English Ed). 2011;64(8):697-712

41. George CJ, Korc B, Ross JS. Appropriate proton pump inhibitor use among older adults: a retrospective chart review. Am J Geriatr Pharmacother. 2008 Dec 1;6(5):249-54 
42. Gurwitz JH, Field TS, Harrold LR, Rothschild J, Debellis K, Seger AC, et al. Incidence and preventability of adverse drug events among older persons in the ambulatory setting. JAMA. 2003 Mar 5;289(9):1107-16.

43. Maher RL, Hanlon J, Hajjar ER. Clinical consequences of polypharmacy in elderly. Expert Opin Drug Saf. 2014;13(1):59.

44. Madhusoodanan S, Bogunovic OJ. Safety of benzodiazepines in the geriatric population. Expert Opin Drug Saf. 2004 Sep 23;3(5):485-93.

45. Morgan SG, Weymann D, Pratt B, Smolina K, Gladstone EJ, Raymond C, et al. Sex differences in the risk of receiving potentially inappropriate prescriptions among older adults. Age Ageing. 2016 Jul;45(4):535-42.

46. Lopez AD, Collishaw NE, Piha T. A descriptive model of the cigarette epidemic in developed countries. Tob Control. 1994 Sep 1;3(3):242-7.

47. Huber CA, Szucs TD, Rapold R, Reich O. Identifying patients with chronic conditions using pharmacy data in Switzerland: an updated mapping approach to the classification of medications. BMC Public Health. 2013;13(1).

48. Ibarra-Castillo C, Guisado-Clavero M, Violan-Fors C, Pons-Vigués M, LópezJiménez T, Roso-Llorach A. Survival in relation to multimorbidity patterns in older adults in primary care in Barcelona, Spain (2010-2014): a longitudinal study based on electronic health records. J Epidemiol Community Health. $2018 \operatorname{Jan} 12 ; 72(3): 185-92$

\section{Publisher's Note}

Springer Nature remains neutral with regard to jurisdictional claims in published maps and institutional affiliations.

Ready to submit your research? Choose BMC and benefit from:

- fast, convenient online submission

- thorough peer review by experienced researchers in your field

- rapid publication on acceptance

- support for research data, including large and complex data types

- gold Open Access which fosters wider collaboration and increased citations

- maximum visibility for your research: over $100 \mathrm{M}$ website views per year

At BMC, research is always in progress.

Learn more biomedcentral.com/submissions 